\title{
Primers for the amplification of the MHC II beta chain exon 2 in the Atlantic goliath grouper (Epinephelus itajara)
}

\author{
Silva-Oliveira Glaucia C. ${ }^{1}$, Silva Anete B. C. ${ }^{1}$, Blanchard Fabian ${ }^{2}$, Nunes Zelia ${ }^{1}$, Torres Rodrigo A. ${ }^{3}$, \\ Sampaio Iracilda ${ }^{1}$, Vallinoto Marcelo ${ }^{1,4,{ }^{*}}$
}

${ }^{1}$ Fed Univ Para, BR-68600000 Braganca, Para, Brazil.

2 IFREMER, Issy Les Moulineaux, France.

${ }^{3}$ Univ Fed Pernambuco, Dept Zool, Ctr Ciencias Biol, Lab Genom Evolut \& Ambiental, Recife, PE, Brazil.

${ }^{4}$ Univ Porto, Ctr Invest Biodiversidade \& Recursos Genet, CIBIO UP, P-4485661 Vairao, Portugal.

* Corresponding author : Marcelo Vallinoto, email address : mvallino@ufpa.br

\begin{abstract}
:
In the present study we designed a pair of primers to amplify the exon 2 of the MHC II beta chain of the Atlantic goliath grouper, which is responsible for the recognition of pathogenic molecules and the regulation of the immune system. Future analyses of this region may provide an important database to understand the evolutionary processes affecting the populations of the goliath grouper, and to predict the conservation perspectives in the species.
\end{abstract}

Keywords : Epinephelus itajara, Genetic diversity, MhcEit-DAB

The formation of spawning aggregations in fishes presents opportunities for efficient fishing and removal of significant proportions of a population within short time frames, however such practice might impact negatively these populations, collapsing them through overexploitation (Sadovy and Eklund, 1999; Tobin et al, 2013). Collapsed populations are likely to reduce their evolutionary fitness towards changes in the environment, eg the raise of new pathogens, making those more susceptible to illnesses

(Eizaguirre and Lenz, 2010).

Family Epinephelidae is an example of collapsed populations in a global scale, due to its directioned fisheries activities to the spawning aggregations (Sadovy de Mitcheson et al, 2012). The goliath grouper (Epinephelus itajara, Lichtenstein, 1822) is the largest bony fish and it is found in the tropical Atlantic Ocean, reaching $25 \mathrm{~m}$ in length and over $400 \mathrm{~kg}$ in weight (Sadovy and Eklund, 1999). As a prominent sport angling species, which is also targeted by commercial fishermen, $E$. itajara has been exploited intensively over the past 30 years, and its populations are now in sharp decline (Aguilar-Perera et al, 2009; McClenachan, 2009). The docile behavior, slow growth, formation of spawning aggregations and current genetic data (low variation) seem to 
make its populations truly vulnerable to extinction (Frias-Torres 2006; Gerhardinger et al. 2006; Koenig et al. 2007; Silva-Oliveira et al. 2008; Mann et al. 2009).

The genes responsible for the recognition of peptides molecules and the regulation of the immune system form part of the Major Histocompatibility Complex (MHC), which is the most polymorphic region of the vertebrate genome (May and Beebee, 2009). The region of the MHC, which recognizes and binds to antigens (Peptide Binding Region- PBR), is located in MHC class II. This region of the genome is responsible for increasing the host's capacity to identify specific invasive agents (Eizaguirre and Lenz, 2010). Therefore, many molecular studies of vertebrates have focused on the amplification of exon 2 of the DQB genes of the $\beta$ chain of the molecules of MHC class II, given the importance of this sequence for the immune response and its high degree of polymorphism (Sonsthagen et al, 2014; He et al, 2014).

No data are available on the attempt to the diversity and selection of MHC II- $\beta$ locus in endangered E. itajara. These new data may provide an important database to understand the evolutionary processes affecting the populations of the E. itajara, and to predict the conservation perspectives in the species, especially in terms of genetic variability.

The samples analyzed were obtained from four locations along the Atlantic in Brazil: Pará (14), Piauí (08), Rio Grande do Norte (10) and Pernambuco (09). The samples of the tissue and fins were obtained from local fishermen between 2000-2008. Total DNA was isolated from muscles and fins, following the method described by Sambrook et al (1989). These samples were amplified using the Polymerase Chain Reaction (PCR) following the protocols of Silva-Oliveira et al. (2013), with an annealing temperature of $54^{\circ} \mathrm{C}$. 
The primers set were based on sequences available for E. coioides, E. akaara

(GU992890-EU399183) and anchored in intron 1, MHC_P0F (5'-

TCAATACAGAGTTGGGCTG-3'), and in the region between exon 2 and intron 2,

MHC_P2R (5'-AACGTTGTTCACACAGACCCTCTC-3'), which favored the isolation of the exon by the PCR technique. Posteriorly two new pairs of primers were designed to access the locus. The amplicons were then sequenced and specific primers were designed for the exon 2- MHC_FOR2 (5'-TTTGTTCCTCAGATGGATTTC-3') and MHC_REV (5'-TTGTTCACACAGACCCTCTCCTTCTC-3'). All the samples were sequenced using these new primers.

The sequences obtained were edited and corrected by Bioedit (Hall, 1999), being posteriously analysed in Mega 5 (Tamura et al. 2011). All the sequences were deposited in the GenBank (xxxxxxxxx).

The name of the locus (MhcEit-DAB) was defined based on the rules proposed by Ellis et al (2006). Exon 2 of the MHC II $\beta$ chain was successfully amplified in 41 samples (13 homozygous), producing a sequence of $198 \mathrm{bp}$ (excluding primers) and 66 aminoacids. The nucleotide sequences of the homozygous included 68 variable sites, 130 conserved sites, and eight singletons. The aminoacid sequences presented 34 conserved sites, 32 variable sites, and two singletons (Table 1). Stop codons were not detected in any of the sequences nor were multiple peaks observed in the chromatograms, both of which indicate that only a single locus was amplified.

Previous genetic data suggested that E. itajara grouper have low genetic diversity even at the most diversified mitochondrial genome region (Silva-Oliveira et al 2008) and the IUCN diagnosis indicates a significant population reduction in E. itajara (http://wwwiucnredlistorg/details/195409/0; accessed in 10/12/2013). Therefore, future 
of the evolutionary processes affecting E. itajara populations and the evaluation and modeling of their evolutionary fitness over the long term.

102

\section{Acknowledgements}

We are grateful to ICMBio and to the Meros do Brasil team for providing the samples of E. itajara, especially the Dr Beatrice Padovani Ferreira. This study was supported by CNPq and FAPESPA (grant 306233/2009-6 to IS, and PRONEX 2007, respectively), and by CAPES and $\mathrm{CNPq}$ stipend. The authors are also grateful to $\mathrm{CNPq}$ for the researcher fellowships provided to RAT, IS and MV.

\section{References}

Aguilar-Perera A, González-Salas C, Tuz-Sulub A, Villegas-Hernández H (2009) Fishery of the goliath grouper, Epinephelus itajara (Teleostei: Epinephelidae) based on local ecological knowledge and fishery records in Yucatan, Mexico. Rev Biol Trop 57: 557-566

Eizaguirre C, Lenz TL (2010) Major histocompatability complex polymorphism: dynamics and consequences of parasite-mediated local adaptation in fishes. J Fish Biol 77: 2023-2047

Ellis SA, Bontrop RE, Antczak DF et al (2006) ISAG/IUIS-VIC Comparative MHC Nomenclature Committee report, 2005. Immunogenetics 57: 953-958

Frias-Torres S (2006) Habitat use of juvenile goliath grouper Epinephelus itajara in the Florida Keys, USA. Endang Species Res 2:1-6 
Gerhardinger LC, Marenzi RC, Bertoncini AA, Medeiros RP, Hostim-Silva M (2006) Local Ecological Knowledge on the goliath grouper Epinephelus itajara (Teleostei: Serranidae) in Southern Brazil. Neotrop Ichthyol 4:441-450

He Y, Xi D, Leng J et al (2014) Genetic variability of MHC class II DQB exon 2 alleles in yak (Bos grunniens). Mol Biol Rep. doi:101007/s11033-014-3071-3

IUCN (2012) International Union for Conservation of Nature, helps the world find pragmatic solutions to our most pressing environment and development challenges Publishing Physics Web http://wwwiucnorg/

Koenig CC, Coleman FC, Eklund AM, Schull J, Ueland J (2007) Mangroves as essential nursery habitat for goliath grouper (Epinephelus itajara). Bull Mar Sci $80: 567-586$

Mann DA, Locascio JV, Coleman FC, Koenig CC (2009) goliath grouper Epinephelus itajara sound production and movement patterns on aggregation sites. Endang Species Res 7:229-236

Sadovy Y, Eklund AM (1999) Synopsis of Biological data on the Nassau Grouper, Ephinephelus striatus (Bloch, 1792), and the Jewfish, E. itajara (Linchtenstein, 1822) NOAA Technical Report NMFS 146:1-68

Sadovy de Mitcheson Y, Craig MT, Bertoncini AA, et al (2012) Fishing groupers towards extinction: a global assessment of threats and extinction risks in a billion dollar fishery. Fish Fish. doi: 101111/j1467-2979201100455x

Sambrook J, Fritsch EF, Maniatis T (1989) Molecular cloning: A laboratory manual. 2nd edn Could Spring Harbor Laboratory Press, New York

Silva-Oliveira GC, Rêgo PS, Schneider H, Sampaio I, Vallinoto M (2008) Genetic characterization of populations of the critically endangered goliath grouper (Epinephelus itajara, Serranidae) from the Northern Brazilian coast. Genet Mol Biol 31: 988-994 
152 Sonsthagen SA, Fales K, Jay CV, Sage GK, Talbot SL (2014) Spatial variation and low

153 diversity in the major histocompatibility complex in walrus (Odobenus rosmarus).

154 Polar Biology. doi: 101007/s00300-014-1450-9

155 Tamura K, Peterson D, Peterson N, Stecher G, Nei M, Kumar S (2011) MEGA5:

156 molecular evolutionary genetics analysis using maximum likelihood, evolutionary

157 distance, and maximum parsimony methods. Mol Biol Evol 28:2731-2739

158 Tobin A, Currey L, Simpfendorfer C (2013) Informing the vulnerability of species to

159 spawning aggregation fishing using commercial catch data. Fish Res 143:47-56. 
Aminoacid

teno buars trav cantor Let caten bencaseva bedarari lipecaste tancases bencastos Epecarse

toeccaros toecearso fencasu

topoury

treasers

\begin{tabular}{|c|c|c|c|c|c|c|c|c|c|c|c|c|c|c|c|c|c|c|c|c|c|c|c|c|c|c|c|c|c|c|c|c|c|c|c|c|c|c|c|c|c|c|c|c|c|c|c|c|c|c|c|c|c|c|c|c|c|c|c|}
\hline & & & & & & & & & & & & & & & 11 & & 12 & & & & & & & & & & & 1 & & & & & & & & & & & & & & & & & & & & & & & & & & & & & & & \\
\hline 1 & & & 4 & 3 & 4 & & $t$ & + & & \pm & 23 & 4 & 3 & 5 & 70 & , & \pm 1 & 2 & 1 & 4 & 5 & 1 & 3 & $t$ & 1 & 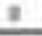 & 1 & 2 & 14 & 5 & 5 & 2 & $t$ & , & a & 1 & 2 & 1 & 43 & 1 & $?$ & $t$ & 1 & 是 & 1 & 2 & 1 & 4 & 3. & $\leqslant$ & 7 & $t$ & , & $t$ & 12 & 1 & 4 & & 4 \\
\hline$\omega$ & & A & 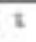 & $\checkmark$ & $\overline{1}$ & c & $\theta$ & 7 & $\mathrm{~N}$ & 5 & 10 & 1 & 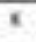 & 0 & 11 & 7 & 10 & 3 & $H$ & 7 & $y$ & $s$ & $\varepsilon$ & i & I & 7 & 1 & $\pi$ & 63 & -1 & 5 & $t$ & 9 & $\varepsilon$ & 7 & $v$ & 6 & 7 & 16 & 7 & 6 & v & $\pi$ & $\bar{N}$ & $A$ & E & 4 & $w$ & N & $v$ & $D$ & 7 & 1 & $\mathrm{~K}$ & & a & & $W^{\prime}$ & 4 \\
\hline$c$ & & 3 & & ' & & & & & & 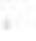 & & & & & & & & & t & & & & & & 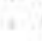 & $i$ & 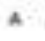 & & & & & $v$ & & & 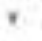 & & & & & 1 & & & 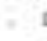 & a & & & 5 & & & r & & 6 & & $\mathrm{t}$ & & 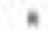 & 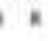 & 1 & \\
\hline 9 & & 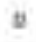 & & & & & $v$ & & & & & , & & & & & & & a & & & & & & & ' & & & & & & $v$ & & & $i$ & & & & & , & & & 7 & a & & & 7 & + & & & & & & 1 & & & $*$ & 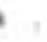 & 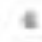 \\
\hline 1 & & 0 & & & . & & $v$ & & & & & , & o & . & 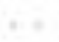 & & $\ldots$ & & $a$ & & & . & & & $=$ & , & & 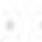 & $\ldots$ & $*$ & & & & & & . & & & 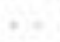 & $r$ & & & . & 0 & & & v & $r$ & . & 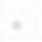 & & & 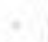 & 1 & & & $*$ & 1. & 0 \\
\hline & & a & & & . & & & & & * & & " & & & & & & & Q & & 1 & & & & & & $v$ & & $\ldots$ & & & & & & & & & &.+ & 1 & & + & . & & 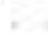 & & $y$ & & & & & & & 1 & & & & a. & \\
\hline & & b & & & & & $v$ & & & , & & 7 & & & & & $\cdots$ & , & a & & & & & & 7 & & $v$ & & & & & & * & & & & . & & 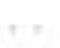 & r & & & & & , & & , & + & & & & & & 1 & $r$ & $n$ & 4 & , & 4 \\
\hline 1 & 6 & $\theta$ & & A & 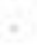 & & $v$ & & & & & " & . & $=$ & 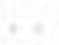 & I & $\therefore$ & & a & & & . & & & & & $v$ & & $\ldots$ & & & & & & & & & & 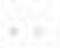 & 1 & & & & & . & & , & 1 & . &. & . & &. & 1 & & & & . & " \\
\hline & 8 & $=$ & & + & . & & $v$ & & & * & & , & & $=$ & & f & & & a & & & . & & & & & $v$ & & $\ldots$ & & & & & & & & & & $\ldots$ & 1 & & * & 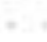 & & . & & N & 1 & & + & & & & i & $\ldots$ & & $*$ & 1. & 6 \\
\hline & & a & & $t$ & & & $v$ & & & 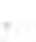 & $\ldots$ & , & 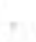 & & & 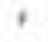 & r & . & & & & & . & & . & & $\boldsymbol{A}$ & & & & & & $=$ & & & & & & $\cdots$ & 1 & & & & & . & & 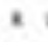 & I & & & & & & 1 & & . & & . & $\tau$ \\
\hline 9 & $c$ & 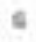 & & A. & & & $v$ & & & & & 7 & & & 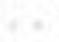 & t & & $=$ & t & & & & & & 4 & & $v$ & & & & & & & & & & & & & f & & & & & & & 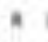 & I & & & & & & 1 & & & & . & a \\
\hline 1 & $c$ & $a$ & & A & $=$ & & $v$ & & & $*$ & & F & & . & & I & $\approx 1$ & & t & & & . & & & . & & $v$ & & $\ldots$ & & & & 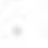 & & & & & & $\cdots$ & F & & & . & & & & $*$ & i & & . & & & & 1 & & & & . & a \\
\hline & & 0 & & & & & $v$ & & & ? & $\ldots$ & " & & & & & $\cdots$ & $=$ & a & & t & & & & r & $i$ & & & & & & & $=$ & & T & & 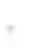 & & $\cdots$ & 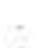 & & & . & 9 & & & $*$ & 2 & & 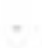 & & & & 1 & & ' & $\checkmark$ & a & E \\
\hline 1 & 6 & 6 & & $A$ & & & $v$ & & & & & , & & & 4. & & & & a & & , & & & & & & $\mathrm{v}$ & & & & & & & & & & & & & I & & & & & . & & $w$ & i & & & & & & I & & & & a. & \\
\hline 1 & & e & & A & & & $v$ & & & & & " & & & & 3 & . & & t & & & & & & & & $v$ & & $\ldots$ & 0 & & & . & & & & & & $\ldots$ & , & & & . & a & & & $\pi$ & 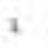 & & $=$ & & & & $v$ & & n & 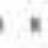 & . & a \\
\hline & $c$ & i & & 4 & & & $v$ & & & & & " & & & & , & & & $t$ & & , & & & & & & v & & & & & & & & & & & & & , & & & & & & & & & & & & & & $v$ & & $*$ & 1 & & \\
\hline
\end{tabular}

